Document downloaded from:

http://hdl.handle.net/10251/56249

This paper must be cited as:

Benítez López, J.; Hueso Pagoaga, JL.; Martínez Molada, E.; Gimenez Valentin, MH.; Riera Guasp, J. (2013). Some Learning Objects to Explain Kepler s Laws. Computer Applications in Engineering Education. 21:1-7. doi:10.1002/cae.20446.

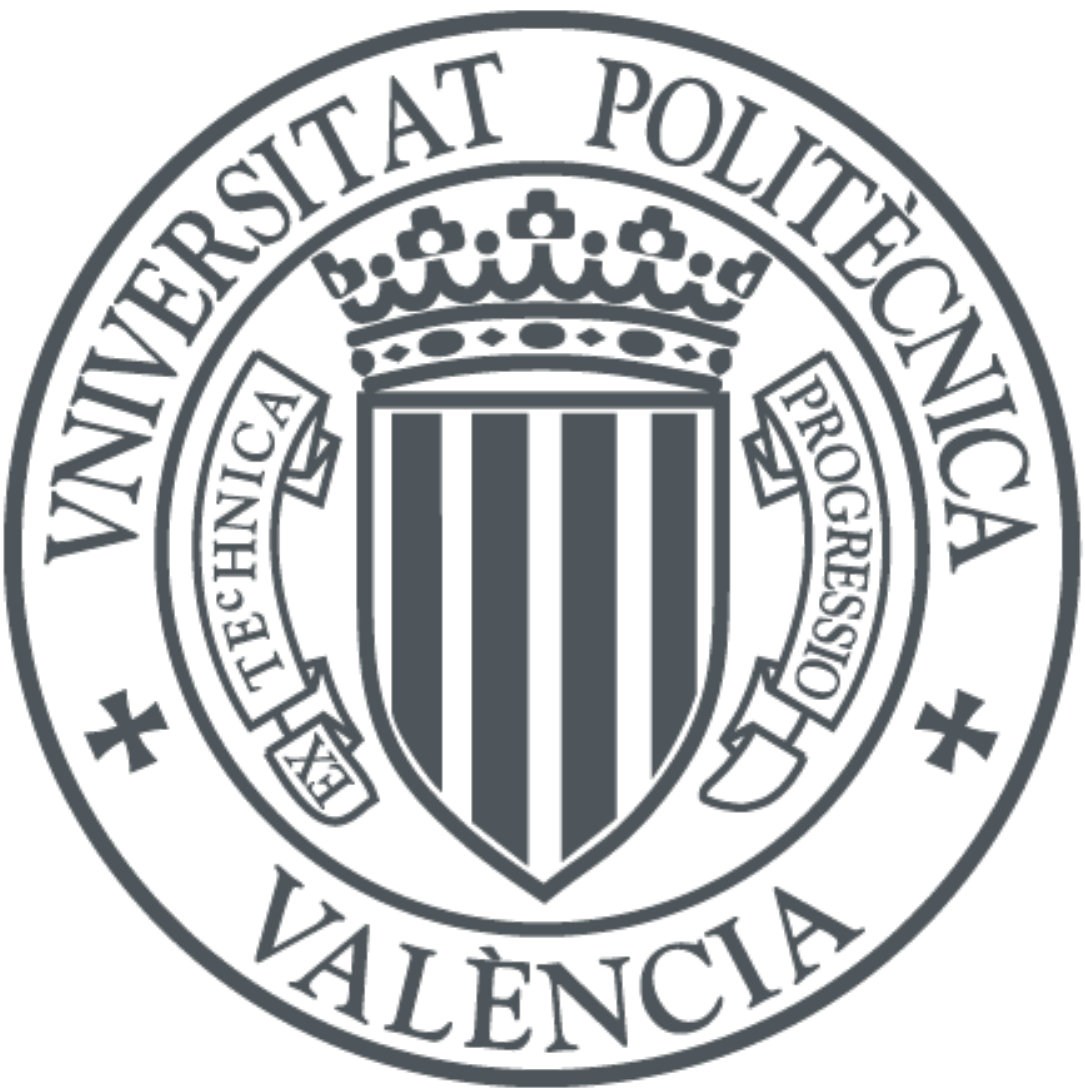

The final publication is available at

http://dx.doi.org/10.1002/cae.20446

Copyright Wiley: 12 months

Additional Information 


\title{
Some learning objects to explain Kepler's laws
}

\author{
Julio Benítez ${ }^{\mathrm{a} *}$ Marcos H. Giménez ${ }^{\mathrm{b} \dagger} \quad$ José L. Hueso ${ }^{\mathrm{b}}$ \\ Eulalia Martínez, ${ }^{\mathrm{b} \S} \quad$ Jaime Riera, ${ }^{\mathrm{b}} \boldsymbol{\Phi}$ \\ ${ }^{a}$ Instituto de Matemática Multidisciplinar, \\ b Falta poner la adscripción!!!!! y los correos!!!! \\ Universidad Politécnica de Valencia, Camino de Vera s/n, 46022, Valencia, Spain.
}

\begin{abstract}
In this paper, we present some learning objects for the study of Kepler's laws that graphically show the orbits and the movements of various planets. One of them shows the orbit of a planet from the point of view of a fixed planet, showing that the orbit is quite difficult. No equations differentials are required, but only elementary vector calculus. The learning object has been implemented in Matlab.
\end{abstract}

Keywords: learning object, Kepler's laws, Matlab

\section{Introduction}

Nowadays we have at our disposal many computer programs to explain Mathematics. We believe that the teaching of this branch of Science will improve if we use such programs. On the other hand, Mathematics and Physics have been closely intertwined since ancient times. We consider that it is a serious mistake to teach Mathematics with no physical intuitions. Physics would help in the understanding of mathematics. Unfortunately, the elimination of the natural sciences from the teaching of Maths is usual. To remedy this situation we propose an integration of the teaching of Mathematics and Physics with a historical perspective. As M. Kline [4] said

The use of real and especially physical problems serves not only to motivate mathematics but to give meaning to it... Mathematical concepts arose from such physical situations or phenomena and their meanings were physical for those who created mathematics in the first place. To rob the concepts of their meaning is to keep the rind and to throw away the fruit.

In this paper we present four Matlab files (these files, with minor differences, can be used with GNU Octave, which is a high-level language, mostly compatible with Matlab, and also

\footnotetext{
${ }^{*}$ Corresponding Author. Email: jbenitez@mat.upv.es

${ }^{\dagger}$ Email: correo@correo.upv.es

${ }^{\ddagger}$ Email: jlhueso@mat.upv.es

$\S$ Email: eumarti@mat.upv.es

"Email: correo@correo.upv.es
} 
freely software). The main purpose of these files is to show graphically and kinematically the three Kepler's laws of planetary motion. Meantime, we provide the mathematical and physical background for the sake of completeness and aiming to show an example of how Mathematics and Physics are inextricable linked. This required background is elementary vector calculus and we do not need know anything about differential equations.

Throughout this paper, vectors of $\mathbb{R}^{3}$ are written by using bold font, the position vector is denoted by $\mathbf{r}(t)$ or simply $\mathbf{r}$, where $t$ is reserved for time. Furthermore, the derivative of any function $f$ with respect to $t$ is denoted by $f^{\prime}$. If $\mathbf{u}$ and $\mathbf{v}$ are vectors in $\mathbb{R}^{3}$, then $\|\mathbf{u}\|$, $\mathbf{u} \cdot \mathbf{v}$, and $\mathbf{u} \times \mathbf{v}$ denote, respectively, the norm (modulus) of $\mathbf{u}$, the scalar (dot) product of $\mathbf{u}$, $\mathbf{v}$, and the vector (cross) product of $\mathbf{u}, \mathbf{v}$. Finally, given a $3 \mathrm{D}$ nonzero vector $\mathbf{u}$, the symbol $\widehat{\mathbf{u}}$ denotes the unitary vector $\mathbf{u} /\|\mathbf{u}\|$.

\section{Historical Background}

The movements of the Sun and the Moon, seen from the Earth, can be described (approximately) as circular orbits. However, there are deviations already observed by the ancient Greeks. Furthermore, the movements of the planets are even more complex. The first mathematical scheme was proposed by Eudoxus (408 BC - 355 BC) in where a rather complicated set of 27 imaginary spheres describes the movements of the Sun, the Moon, and the planets. Aristarchus of Samos (about 310 BC - about 230 BC) proposed a heliocentric model of the solar system; but this model had no influence. The first great attempt to describe celestial mechanics was made by Ptolemy (about 85 - about 165). In its "Almagest", he propounded the geocentric theory in a form that prevailed for 1400 years until Copernicus $(1473-1543)$ presented his heliocentric theory in "De revolutionibus orbium colestium".

One of the most important advances of the physical sciences after the medieval ages was noting that we must mathematically describe the nature and not asking why does the nature behave in a concrete way. Kepler $(1571-1630)$ did so in describing the solar system in his very famous three (empirical) laws:

Law I The path of a planet is an ellipse being the Sun one focus.

Law II An imaginary line drawn from the Sun to the planet sweeps out equal areas in equal intervals of time.

Law III The ratio of the squares of the periods of any two planets is equal to the ratio of the cubes of their average distances from the sun (being the orbit closed, the planet will return to its starting point in a time $T$ called the period of the orbit).

From Kepler's laws, Newton $(1643$ - 1727) was able to deduce the law of universal gravitation or the inverse-square law, i.e., all matter attracts all other matter with a force proportional to the product of their masses and inversely proportional to the square of the distance between them. As a suggested lecture, we recommend [1].

Why did Kepler state his laws in this way? This question is not dealt in textbooks and we want to answer it with more detail. The first law is a geometrical law: it tells us about the form of the orbit, but not about how the planet moves. The second law is a kinematical law: it tells us about the velocity of the planet or how the planet moves; at a first sight, this law is strange because the scalar velocity is not mentioned, unlike the areal velocity. Why? 
As we will see, in Kepler's time, the scalar velocity was impossible to be exactly calculated, and this impossibility forced Kepler to state his second law in that manner. The third law gives us a scale of the solar system: let $a$ be the average distance of the Earth to the Sun; since the period of the Earth is, obviously, 1 year, we have $T_{p}^{2 / 3}=a_{p} / a$ where $T_{p}$ is the period of another planet (a number easy to find) easy and $a_{p}$ is the average distance of this same planet to the Sun.

\section{Mathematical and Physical Background}

This is not the place to explain the main mathematical and physical developments of the Astronomy (there are hundred of textbooks on vector calculus or physics explaining this branch of the Science. For a comprehensive text we can cite [5]). As we pointed out in the introduction, physical sciences can not be developed without mathematics, and mathematics can be better explained by means of physical models. Thus, we will try to link these scientific disciplines. At the same time, in our opinion, it is important to stress the kinematical models in contraposition the statical models.

Let us fix a coordinate system with the sun at the origin. Newton's laws leads to study the vector equation

$$
m \mathbf{r}^{\prime \prime}=-\frac{k}{\|\mathbf{r}\|^{2}} \widehat{\mathbf{r}} .
$$

As is well known, the constant $k$ depends on the kind of the force, and it describes the strength of the force. For Newton's law of gravitation, one has $k=G M m$, where $G$ is a universal constant, $M$ is the mass of the Sun, and $m$ is the mass of the planet.

Let us make a parenthesis by studying (1) assuming that the orbit is a circumference (such situation is not academic, it happens when some artificial satellites orbit around the Earth). This study is so simple that it is worthy to present it to any student of vector calculus. Let $\mathbf{r}(t)=R(\cos \theta(t), \sin \theta(t))$ be the orbit, being $R$ its radius. We get $\mathbf{r}^{\prime \prime}=R \theta^{\prime \prime} \widehat{\boldsymbol{\theta}}-R\left(\theta^{\prime}\right)^{2} \widehat{\mathbf{r}}$, where $\widehat{\boldsymbol{\theta}}=(-\sin \theta, \cos \theta)$. From (1) one has $\theta^{\prime \prime}=0$ and $m R\left(\theta^{\prime}\right)^{2}=k / R^{2}$. From the first relation we obtain that $\theta^{\prime}$ is a constant, say $\omega$; i.e., the satellite has a uniform circular movement with angular velocity $\omega$. This is a simplified version of the Kepler's second law. From the second relation one gets $R^{3} \omega^{2}=G M$, and this relation is a particular case of Kepler's third law.

For the sake of completeness, we will deduce Kepler's laws by using vector methods (see, for example, [1, Ch. 6] or [3, Ch. 9] for another deduction). In fact, Kepler's first law is not exactly correct: ellipses are the true shape of an orbit only for two isolated masses; two masses orbit around their center of mass, which is at a focus of their elliptical orbits; and unbounded orbits are described by other conic sections (parabolas and hyperbolas).

\subsection{Kepler's second law}

From (1) and having in mind that $\mathbf{r} \times \widehat{\mathbf{r}}=\mathbf{0}$, we get $\left(\mathbf{r} \times \mathbf{r}^{\prime}\right)^{\prime}=\mathbf{r} \times \mathbf{r}^{\prime \prime}=\mathbf{0}$, and thus, $\mathbf{r} \times \mathbf{r}^{\prime}$ is a constant vector. The vector $\mathbf{L}=\mathbf{r} \times\left(m \mathbf{r}^{\prime}\right)$ is called the angular momentum of the planet, and therefore, the directed area $\mathbf{A}=\mathbf{A}(t)$ swept out by $\mathbf{r}$ is given by

$$
\mathbf{A}(t)=\frac{1}{2} \int_{t_{0}}^{t} \mathbf{r}(\tau) \times \mathbf{r}^{\prime}(\tau) \mathrm{d} \tau=\frac{\left(t-t_{0}\right)}{2 m} \mathbf{L}
$$

This is second Kepler's law (parenthetically, we can deduce that $\mathbf{r} \cdot \mathbf{L}=0$, which means that the orbit of the planet is planar). 


\subsection{Kepler's first law}

From $\mathbf{r}^{\prime}=(\|\mathbf{r}\| \widehat{\mathbf{r}})^{\prime}=\|\mathbf{r}\|^{\prime} \widehat{\mathbf{r}}+\|\mathbf{r}\| \widehat{\mathbf{r}}^{\prime}$ we get $\mathbf{L}=m \mathbf{r} \times \mathbf{r}^{\prime}=m\|\mathbf{r}\|\left(\mathbf{r} \times \widehat{\mathbf{r}}^{\prime}\right)$. Observe that $\widehat{\mathbf{r}} \cdot \widehat{\mathbf{r}}^{\prime}=0$ because $\widehat{\mathbf{r}} \cdot \widehat{\mathbf{r}}$ is constant. Now (1) leads to

$$
\mathbf{r}^{\prime \prime} \times \mathbf{L}=m\|\mathbf{r}\|\left[\mathbf{r}^{\prime \prime} \times\left(\mathbf{r} \times \widehat{\mathbf{r}}^{\prime}\right)\right]=-\frac{k}{\|\mathbf{r}\|}\left[\widehat{\mathbf{r}} \times\left(\mathbf{r} \times \widehat{\mathbf{r}}^{\prime}\right)\right]=-\frac{k}{\|\mathbf{r}\|}\left[\left(\widehat{\mathbf{r}} \cdot \widehat{\mathbf{r}}^{\prime}\right) \mathbf{r}-(\widehat{\mathbf{r}} \cdot \mathbf{r}) \widehat{\mathbf{r}}^{\prime}\right]=k \widehat{\mathbf{r}}^{\prime}
$$

Since $\mathbf{L}$ is constant, we get $\left(\mathbf{r}^{\prime} \times \mathbf{L}-k \widehat{\mathbf{r}}\right)^{\prime}=\mathbf{0}$. Therefore, there exists $\mathbf{w} \in \mathbb{R}^{3}$ such that $\mathbf{r}^{\prime} \times \mathbf{L}=k \widehat{\mathbf{r}}+\mathbf{w}$. Hence

$$
\left(\mathbf{r}^{\prime} \times \mathbf{L}\right) \cdot \mathbf{r}=(k \widehat{\mathbf{r}}+\mathbf{w}) \cdot \mathbf{r} .
$$

The left side of (3) reduces to $\left(\mathbf{r}^{\prime} \times \mathbf{L}\right) \cdot \mathbf{r}=\left(\mathbf{r} \times \mathbf{r}^{\prime}\right) \cdot \mathbf{L}=(\mathbf{L} \cdot \mathbf{L}) / m=\|\mathbf{L}\|^{2} / m$. The right side of (3) becomes $(k \widehat{\mathbf{r}}+\mathbf{w}) \cdot \mathbf{r}=k\|\mathbf{r}\|+\|\mathbf{w}\|\|\mathbf{r}\| \cos \theta=\|\mathbf{r}\|(k+\|\mathbf{w}\| \cos \theta)$, being $\theta$ the angle between $\mathbf{r}$ and $\mathbf{w}$. Hence (3) leads to

$$
\|\mathbf{r}\|=\frac{\frac{\|\mathbf{L}\|^{2}}{k m}}{1+\frac{\|\mathbf{w}\|}{k} \cos \theta} .
$$

This is the equation of a conic with eccentricity $e=\|\mathbf{w}\| / k$. Recall that if $e>1$ then the orbit is an hyperbola, if $e=1$ then the orbit is a parabola, and if $0 \leq 1$ then the orbit is an ellipse.

In the sequel, we will suppose that the orbit of the planet under consideration is an ellipse (see Fig. 1). Let $\widehat{\mathbf{i}}$ and $\widehat{\mathbf{j}}$ be the two unitary vectors depicted in Fig. 1, and $\widehat{\mathbf{k}}=\widehat{\mathbf{i}} \times \widehat{\mathbf{j}}$. Let $a$ be the semimajor axis and $b$ the semiminor axis (with $b \leq a$ ). It can be proved that $c=\sqrt{a^{2}-b^{2}}$ is the distance between the center of the ellipse and any of the two focus. Furthermore, the eccentricity $e$ is defined by $e=c / a$ (observe that when $e=0$, the ellipse becomes a circumference). It is a simple textbook exercise of vector calculus to prove that the area of such ellipse is $\pi a b$.

Since the orbit of the planet is closed, the planet will return to its starting point in a time $T$ called the period of the orbit. From (2) it follows

$$
\pi a b \widehat{\mathbf{k}}=\frac{T}{2 m} \mathbf{L} .
$$

It is necessary to be able to locate the planet at any given time $t$ and the second Kepler's law will be the main tool to this end. Let us remark that the problem of the localization of the planet is not developed in the standard textbooks; thus we will study it.

The orbit of the planet can be described by $\mathbf{x}=a \cos \phi \widehat{\mathbf{1}}+b \sin \phi \widehat{\mathbf{J}}$, where $\phi=\phi(t)$. (parenthetically, it is a common mistake among the students saying that $\phi$ is the angle formed by $\mathbf{x}$ and $\widehat{\mathbf{i}}$ ). Now, we have

$$
\mathbf{r}=-c \widehat{\mathbf{i}}+\mathbf{x}=-a e \widehat{\mathbf{i}}+a \cos \phi \widehat{\mathbf{l}}+b \sin \phi \widehat{\mathbf{J}}=a(-e+\cos \phi) \widehat{\mathbf{i}}+b \sin \phi \widehat{\mathbf{j}},
$$

hence, $\mathbf{r}^{\prime}=-a \phi^{\prime} \sin \phi \widehat{\mathbf{1}}+b \phi^{\prime} \cos \phi \widehat{\mathbf{J}}$, and thus, after simplifying,

$$
\mathbf{L}=m\left(\mathbf{r} \times \mathbf{r}^{\prime}\right)=m a b \phi^{\prime}(1-e \cos \phi) \widehat{\mathbf{k}} .
$$

Kepler's second law explains why the nearer each planet approaches the Sun, the faster it moves. Observe that (7) explains it in a quantitative way since $\|\mathbf{L}\|$ is constant. Let us remark 


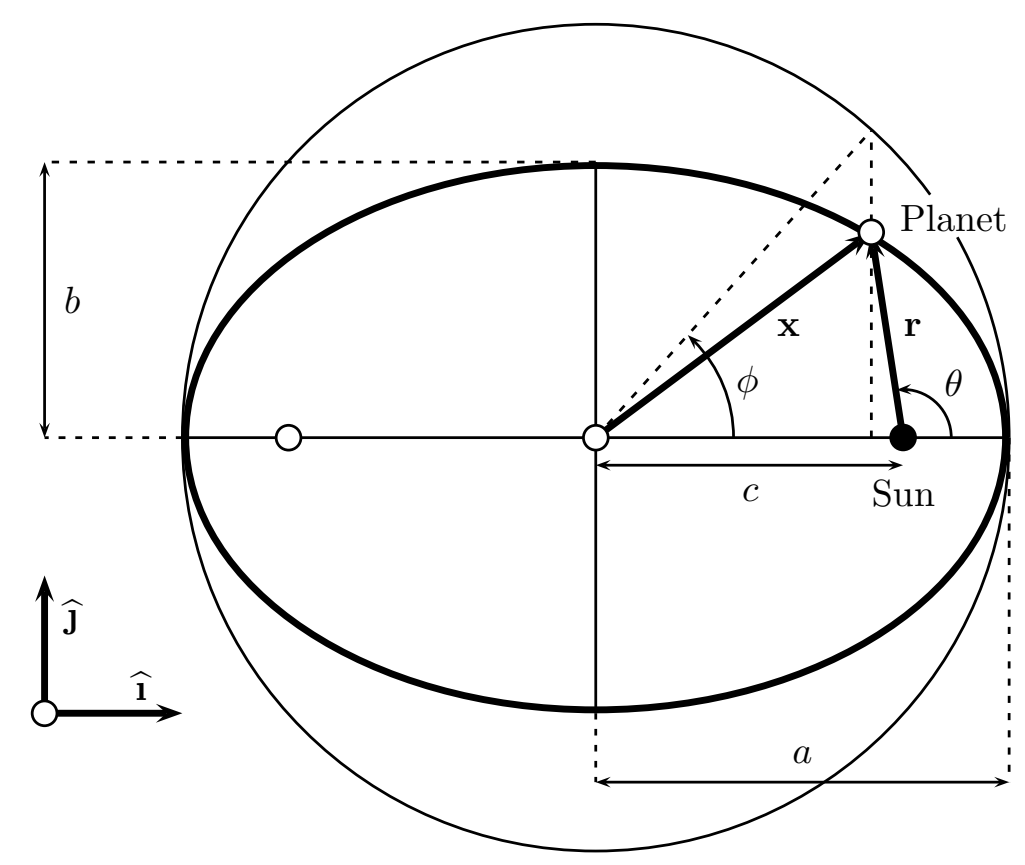

Figure 1: The geometry of the orbit of a planet.

that the scalar velocity satisfies $v=\left\|\mathbf{r}^{\prime}\right\|=\left|\phi^{\prime}\right| \sqrt{a^{2} \sin ^{2} \phi+b^{2} \cos ^{2} \phi}$, and an expression of the form $\int v(t) \mathrm{d} t$ leads to an elliptical integral, which can not be exactly solved.

Having in mind that $\mathbf{L}$ is constant, integrating (7) with respect $t$, and assuming $\phi(0)=$ 0 (i.e., associating the initial time with the position at the point nearest to the center of attraction), we get $t \mathbf{L}=\operatorname{mab}(\phi-e \sin \phi) \widehat{\mathbf{k}}$, and using (5) yields

$$
\frac{2 \pi t}{T}=\phi-e \sin \phi
$$

Since $0 \leq e<1$, for any $t$, there exists a unique $\phi=\phi(t)$ such that (8) holds. This angle $\phi$ enables us to locate the planet at any time $t$. Unfortunately, this equation has not an analytic solution, and therefore, we have to solve it by using non exact methods. Matlab and Octave use the function fzero (Dekker' algorithm is used in both programs; see [2, Ch. 4] for a deep study of this algorithm). But we have another choice: we can use that the values of $e$ in Table 1 are small. To find the angle $\phi$ satisfying (8), let us fix $t$ in (8), consider $\phi$ as a function of $e$, and use a Taylor approximation:

$$
\phi \simeq \frac{2 \pi t}{T}+e \sin \left(\frac{2 \pi t}{T}\right)+\frac{e^{2}}{2} \sin \left(\frac{4 \pi t}{T}\right) .
$$

However, the angle $\theta$ depicted in Figure 1 has a more geometric meaning than $\phi$. Thus, we will express $\theta$ in terms of $\phi$. From (6) and using $b^{2}=a^{2}\left(1-e^{2}\right)$, we get $\|\mathbf{r}\|=a(1-e \cos \phi)$. Now, we can compute $\mathbf{r} \cdot \widehat{\mathbf{1}}$ by using two different ways (one of them uses (6)):

$$
\mathbf{r} \cdot \widehat{\mathbf{i}}=\left\{\begin{array}{l}
\|\mathbf{r}\|\|\hat{\mathbf{1}}\| \cos \theta=a(1-e \cos \phi) \cos \theta, \quad \Longrightarrow \quad \cos \theta=\frac{-e+\cos \phi}{1-e \cos \phi} . \\
a(-e+\cos \phi),
\end{array}\right.
$$

Finally, by making some algebra and using the half-angle formula for the tangent we get 


\begin{tabular}{|c|cccccccc|}
\hline Planet & Mercury & Venus & Earth & Mars & Jupiter & Saturn & Uranus & Neptune \\
\hline$e$ & 0.206 & 0.007 & 0.017 & 0.093 & 0.048 & 0.056 & 0.046 & 0.010 \\
$a$ & 0.3871 & 0.7233 & 1 & 1.5273 & 5.2028 & 9.5388 & 19.1914 & 30.0611 \\
$T$ & 0.2408 & 0.6152 & 1 & 1.8809 & 11.862 & 29.458 & 84.01 & 164.79 \\
\hline
\end{tabular}

Table 1: This table was obtained from [1] and [6]. As a curious remark, let us permit say that in [1] Pluto was still considered a planet. The value of $a$ is measured in astronomical units and $T$ in years.

$$
\tan \left(\frac{\theta}{2}\right)=\sqrt{\frac{1+e}{1-e}} \tan \left(\frac{\phi}{2}\right) .
$$

Since $\phi / 2$ and $\theta / 2$ are always located in the same quadrant, relation (10) allows us to find $\theta$ for a given $\phi$.

\subsection{Kepler's Third law}

Now, we will prove Kepler's Third law. If we assume that the origin of coordinates is located at the center of the ellipse, then the equation of the orbit is $x^{2} / a^{2}+y^{2} / b^{2}=1$ and $\mathbf{r}(\pi / 2)=$ $(c, l)$ for some $l>0$. Hence $c^{2} / a^{2}+l^{2} / b^{2}=1$, which yields $b^{2}=a l$. Now (4) leads to $l=\|\mathbf{r}(\pi / 2)\|=\|\mathbf{L}\|^{2} /(m k)$. Using (5) yields $T^{2}=4 \pi^{2} a^{2} b^{2} m^{2} /\|\mathbf{L}\|^{2}=4 \pi^{2} a^{3} m / k$, or using the value of $k$ we have $T^{2} / a^{3}=4 \pi^{2} / G M$.

\section{$4 \quad$ Files}

In this section we provide the $\mathrm{m}$ files that could serve to explain in a interactive way the three Kepler's laws.

The first file (called $\mathrm{k} 1 \mathrm{~m}$ ) draws the orbit with eccentricity $e \in[0,1[$, locates the planet at any time $t \in[0, T]$ (it is assumed that the planet start at the perihelion), and paints the area swept out by the position vector. If $t \leq T / 2$, the file makes the former but starting at the perihelion and the aphelion. This shows clearly Kepler's second law as is seen in Fig. 2. Also, the file computes the angle(s) swept out by the position vector.

Now we give some explanation: Throughout this file $a=1$ and $T=1$ are assumed. The variable Tempus is reserved for the final time. Lines 12-14 draw the ellipse. In line 15, it is found $\phi$ satisfying $\phi-e \sin \phi=2 \pi$ Tempus. Note that the function fzero and the "inner anonymous function" fkepler are used (alternatively, under the assumption that $e \simeq 0$, we can use (9) in order to make fewer operations). Lines 16-19 paint the area swept out by the position vector. Lines 20-22 compute the angle (in radians) swept out by using (10). We do not explain lines 23-35 because they are completely analogous to the former ones. Observe that if the origin of coordinates is the center of the ellipse, the focuses of this ellipse are located at $\pm(c, 0)$. We plot the sun at $(c, 0)$; but recall that we have assumed $a=1$, hence $c=e$, which explains line 36 .

\footnotetext{
1 function angle $=\mathrm{k} 1$ (e,Tempus)

$2 \% \mathrm{a}=\mathrm{k} 1$ (e,Tempus) draws the orbit a planet
} 


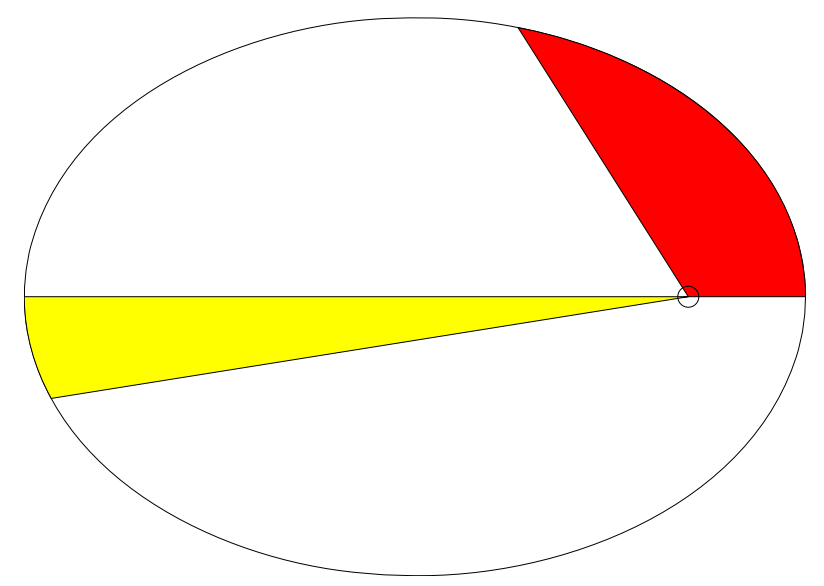

Figure 2: This figure has been obtained by making $k 1(0.7,0.1)$. The output is [122.32 9.07].

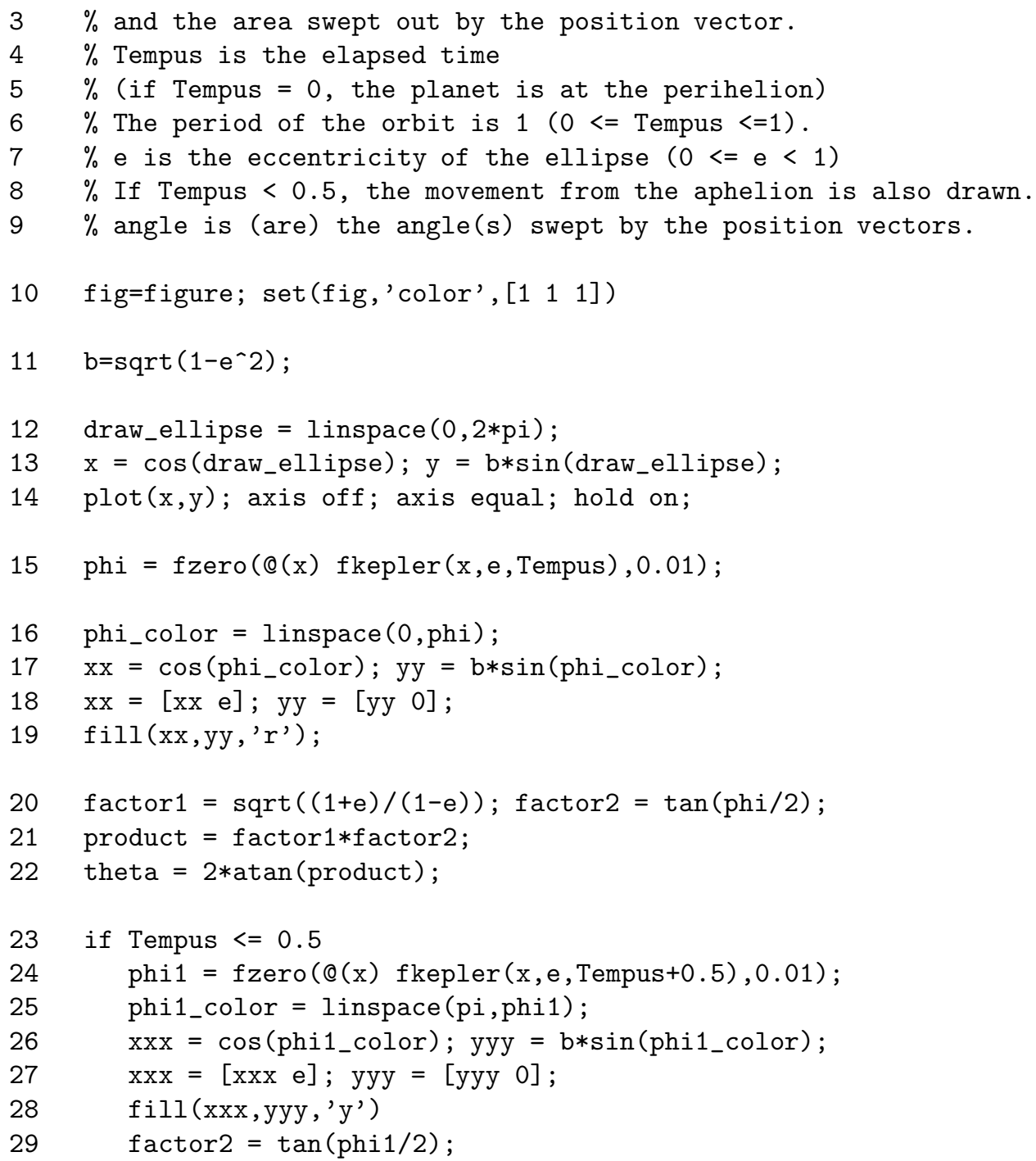




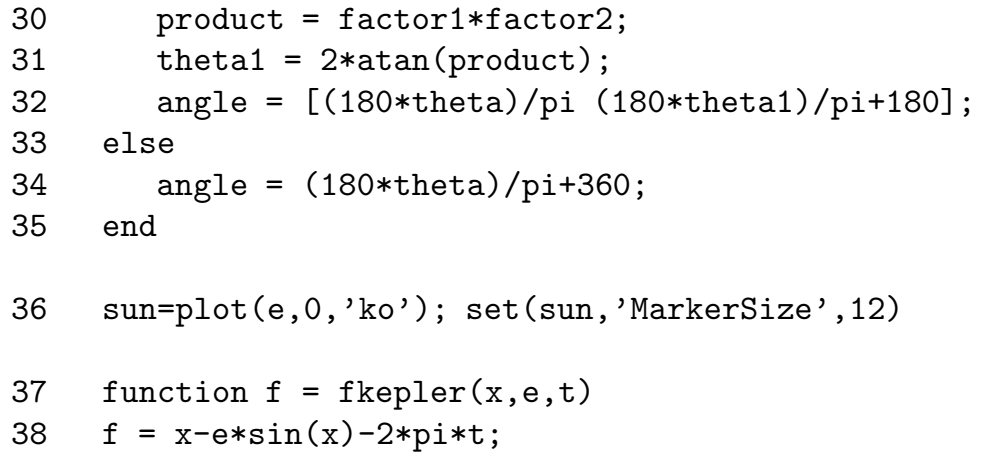

The following file $(\mathrm{k} 2 . \mathrm{m})$ is "more kinematical" because shows the movement of the planet. Observe lines 16-17 and 24-25 in where the planet and its position vector move without using the function delete. This file does not require further explanation because is similar than $\mathrm{k} 1 . \mathrm{m}$.

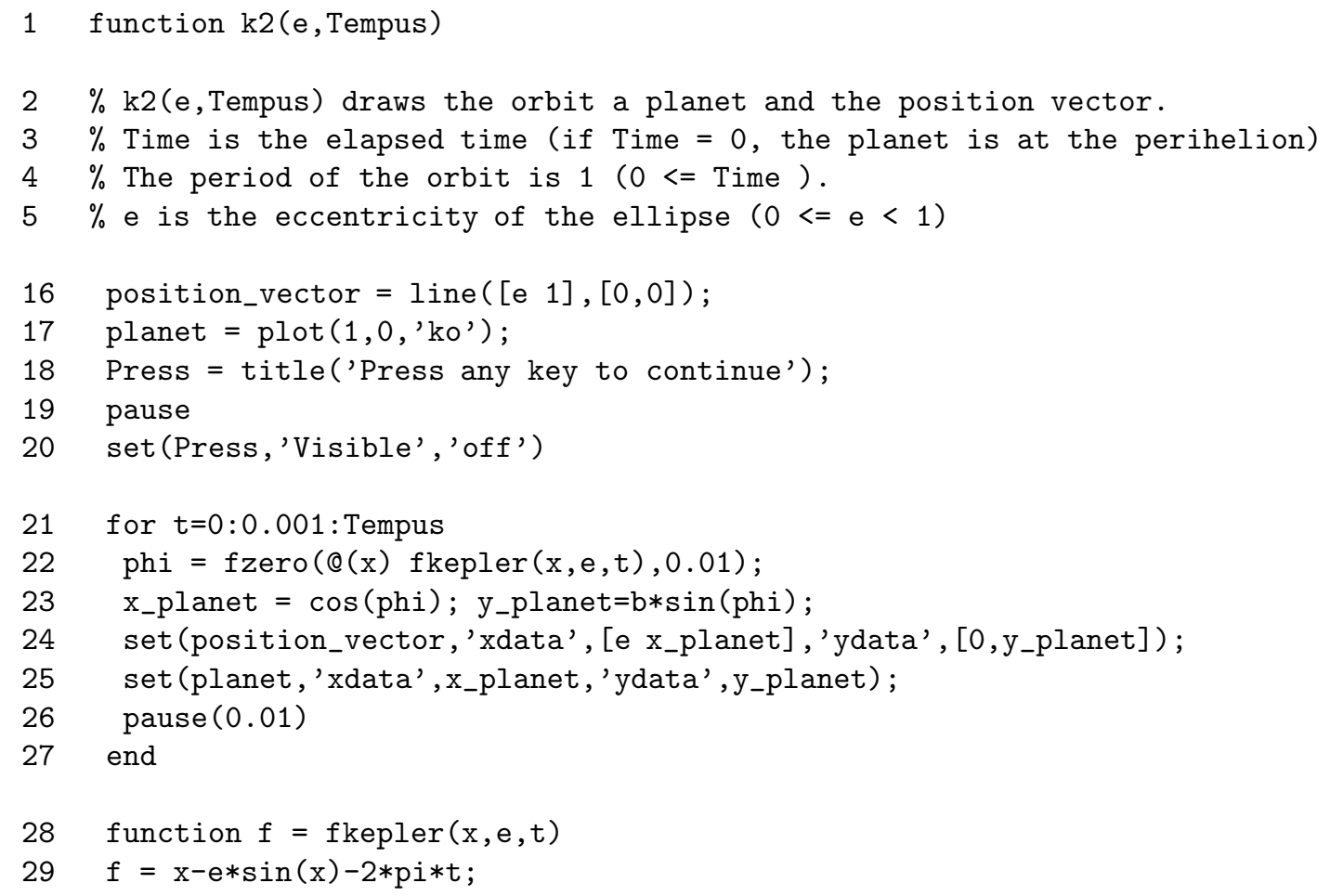

The following two files draw the orbits of two planets in the same solar system. To this end, let us recall that the periods of the orbits must satisfy Kepler's third law. The first file draws the orbits with a fixed sun, whereas the second file draws the trajectories of the Sun and one planet from the point of view of one inhabitant of the another planet.

Let us briefly explain the foregoing file $\mathrm{k} 3$. Kepler's third law is codified in line 15 (it is assumed that the period of one planet is 1). We shall use the polar expression (4) to draw the orbit of the two involved planets with the sun being plotted at the origin (line 14). This expression can be written as $\|\mathbf{r}\|=l /(1+e \cos \theta)$, with the positive number $l$ satisfying $l=b^{2} / a$ (this same number $l$ also appears in subsection 3.3). Line 13 draws the two orbits. Finally, the variable planet_1 stores in lines 16 and 33 the coordinates of the position of one 
planet (the variable planet_2 is analogous). Also the file draws the line joining the sun and the planets (lines 17, 19 and 34, 36)

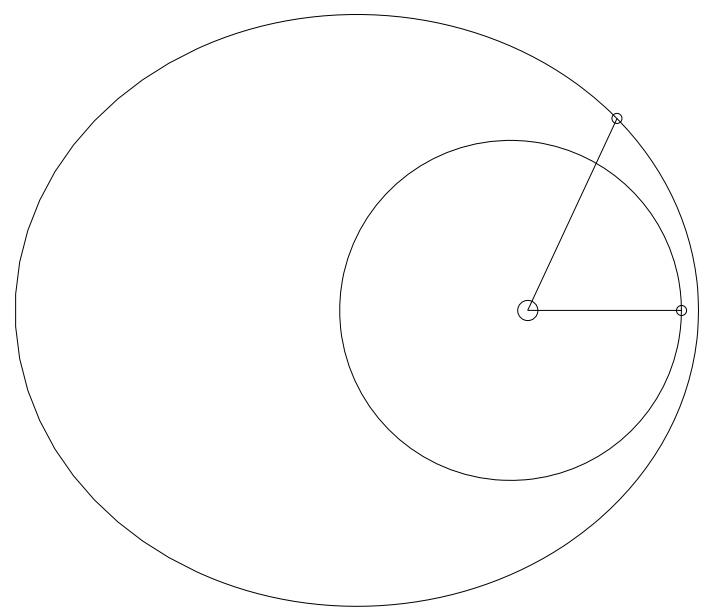

Figure 3: This figure has been obtained by making k3([0.1 0.5], [1 2], 3).

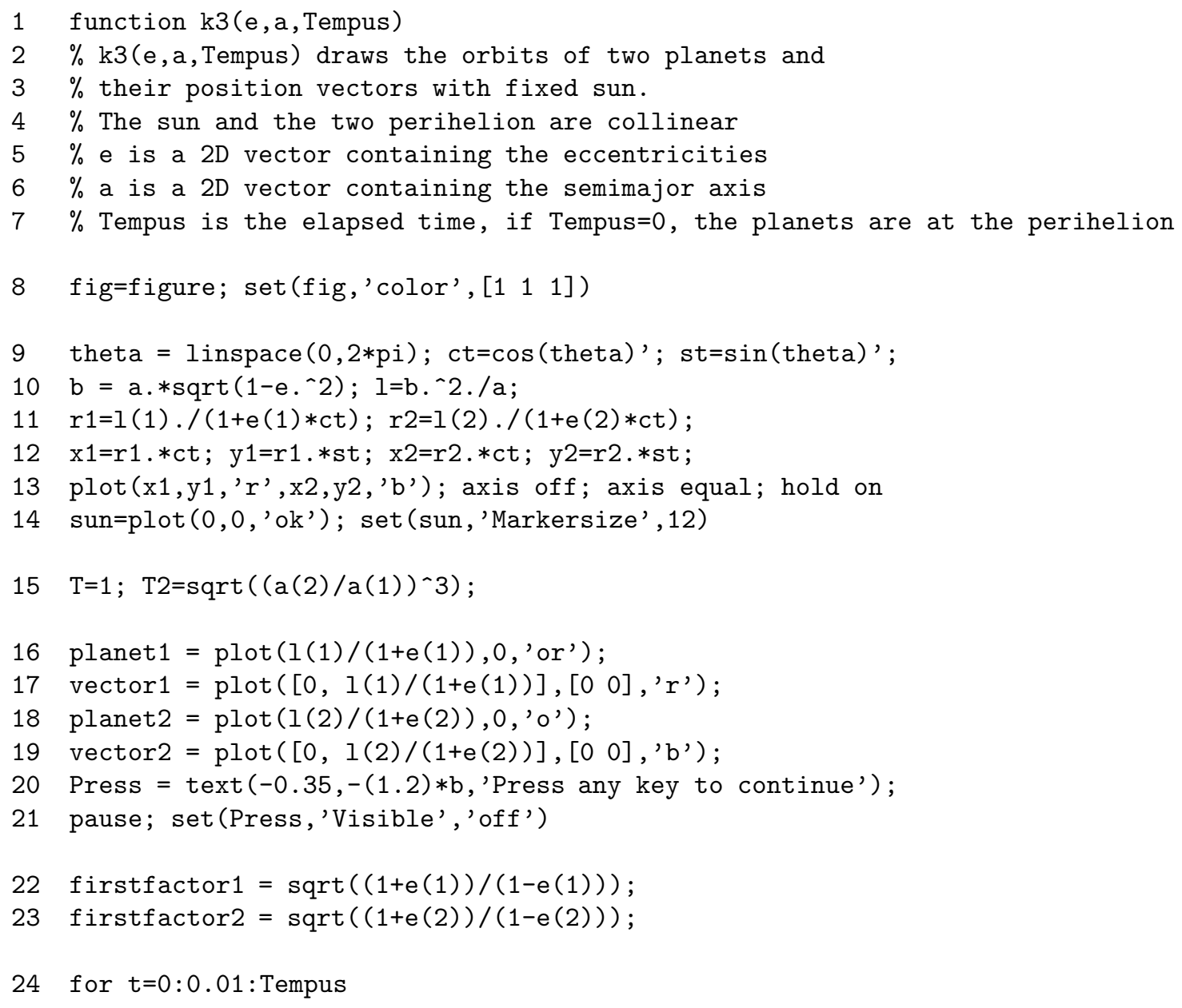




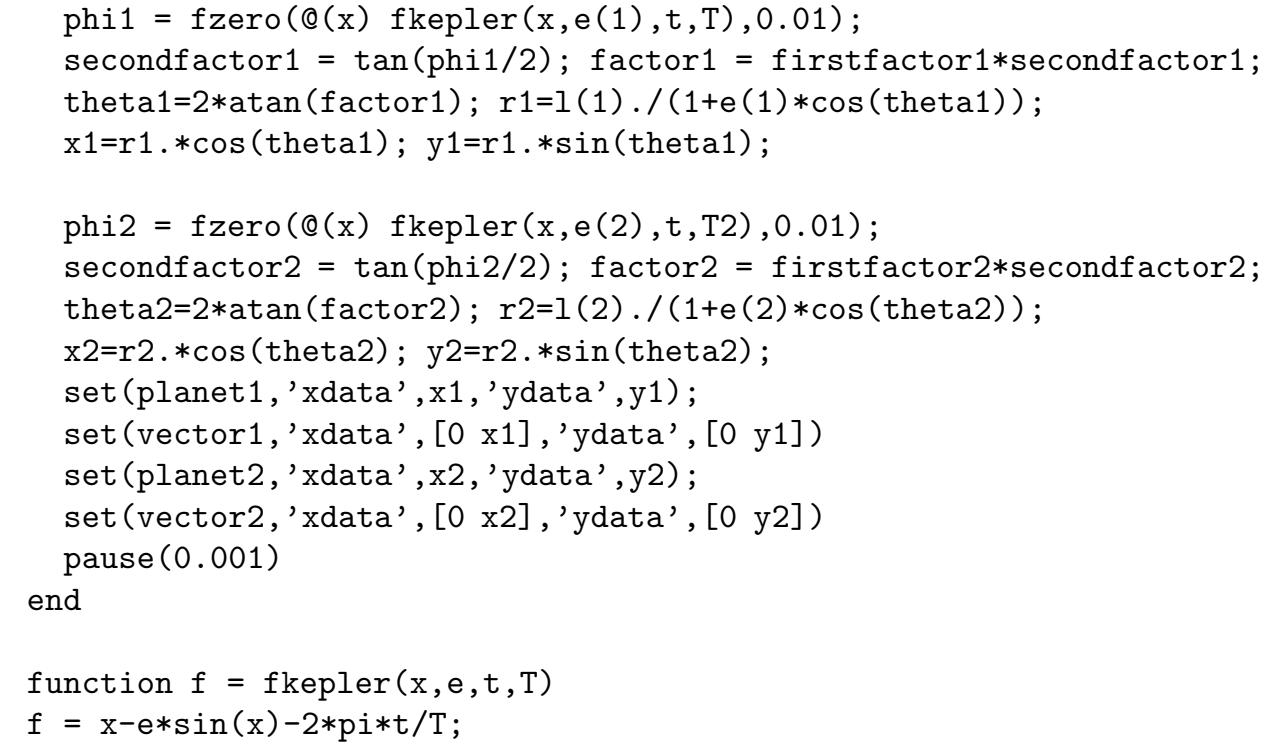

Ancient astronomers observed that Mars generally traveled eastward, except every couple of years, when this planet reversed course, going westward for a few months. Let us remember the system proposed by Ptomely: planets orbit Earth in quasi circular paths; the variations were explained by adding complex sets of circles upon circles. In the $16^{\text {th }}$ century, Copernicus suggested that the movement of Mars could be explained by assuming that all planets (including Earth) orbit around the Sun. Later, Kepler proposed that the orbit were ellipses. How is the movement of Mars seen from the Earth? Seeing this movement helps to admire more the talent of Copernicus and Kepler, because this movement is very different from an ellipse (as is easily seen in Fig. 4, in where the true eccentricities and the semimajor axis of the Earth and Mars were introduced as inputs).

The mathematics inherent of file $\mathrm{k} 4 \mathrm{~m}$ are very similar as in $\mathrm{k} 3 . \mathrm{m}$. There are only two main differences: (a) In order to change the origin coordinates, we make a translation (lines 15-16 and 38-39); and (b) The file does not draw directly the orbit of the non fixed planet, but this orbit is pictured step by step in lines $44-45$ (this is the reason for storing the values of old_sun and old_planet2 in line 27).

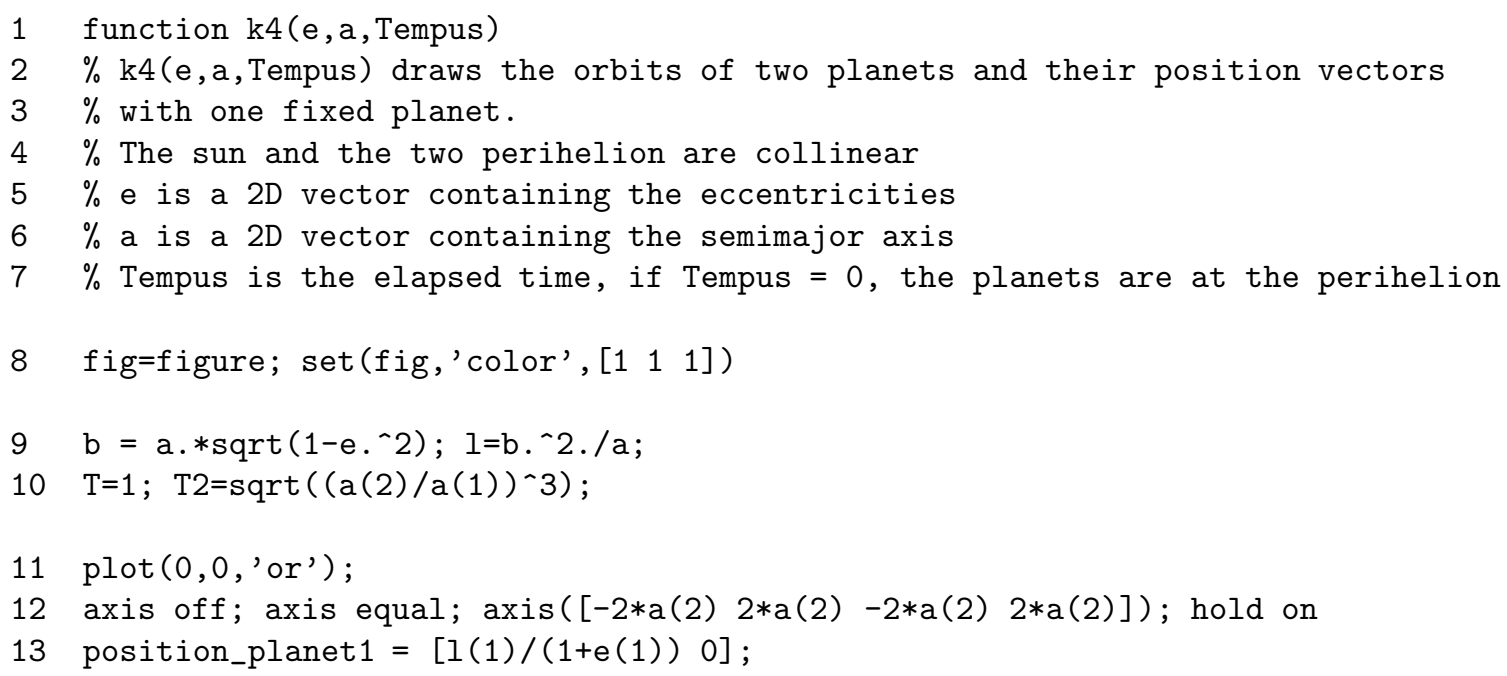




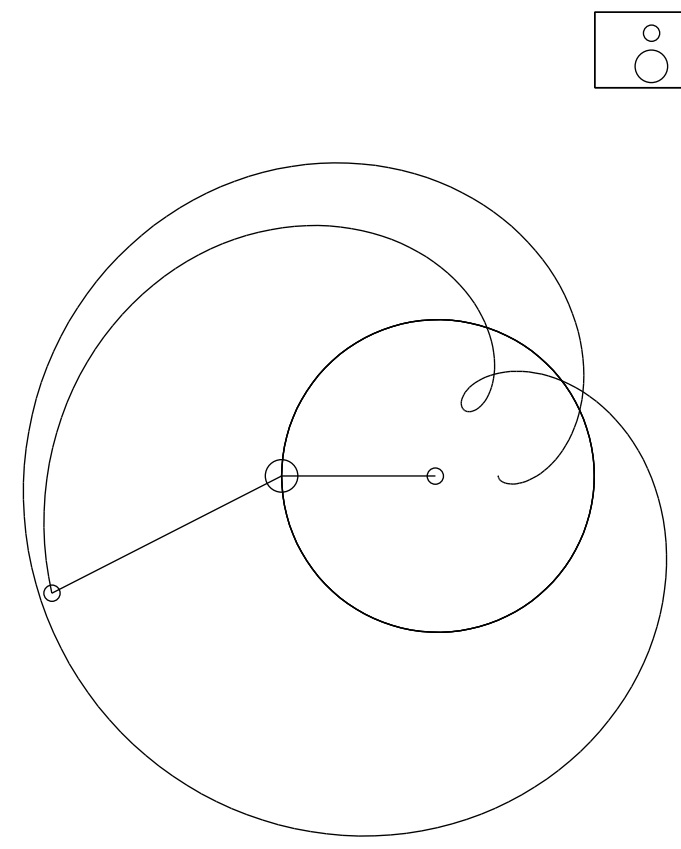

Figure 4: This figure has been obtained by making k4([0.017 0.093], [1 1.5273], 3).

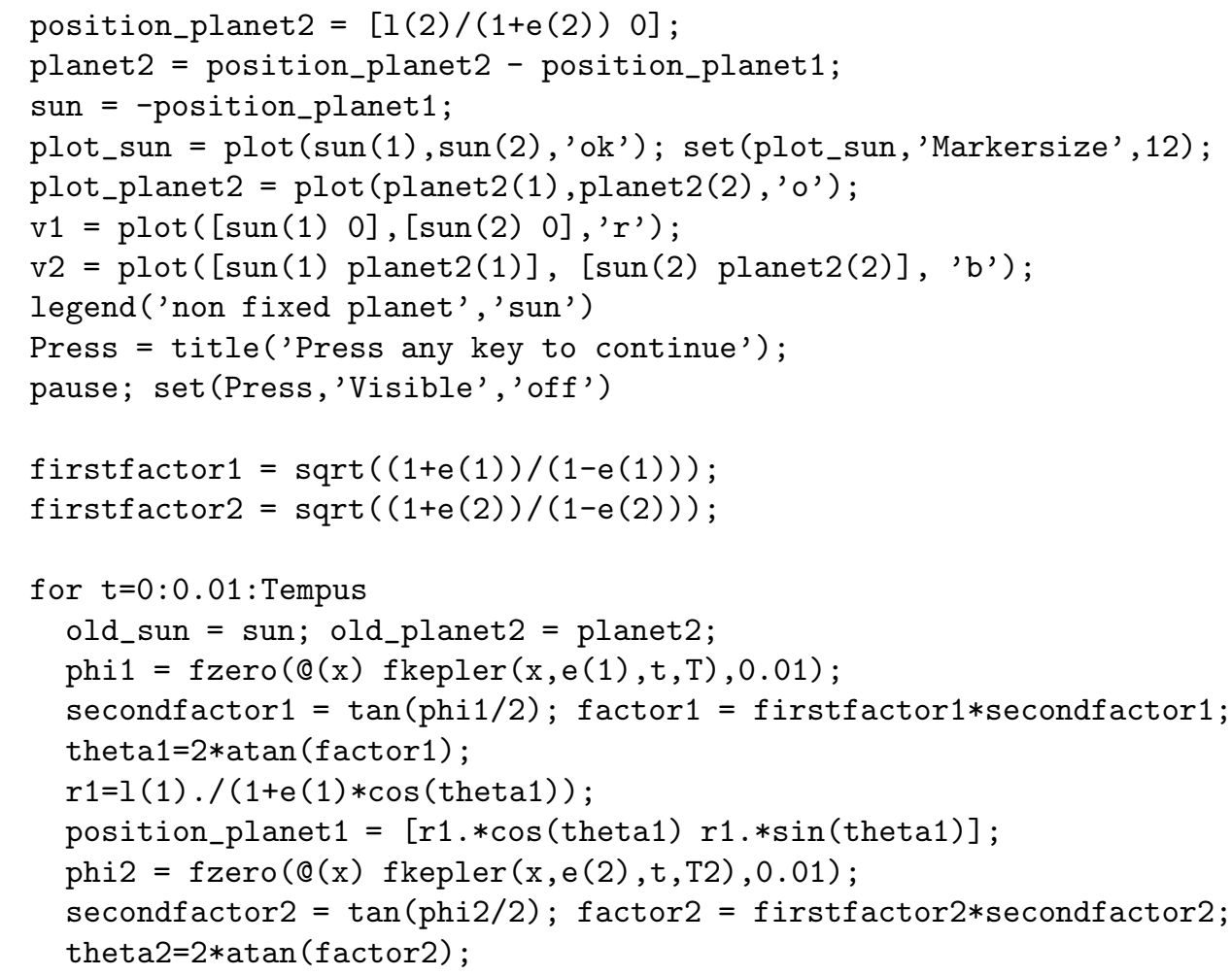




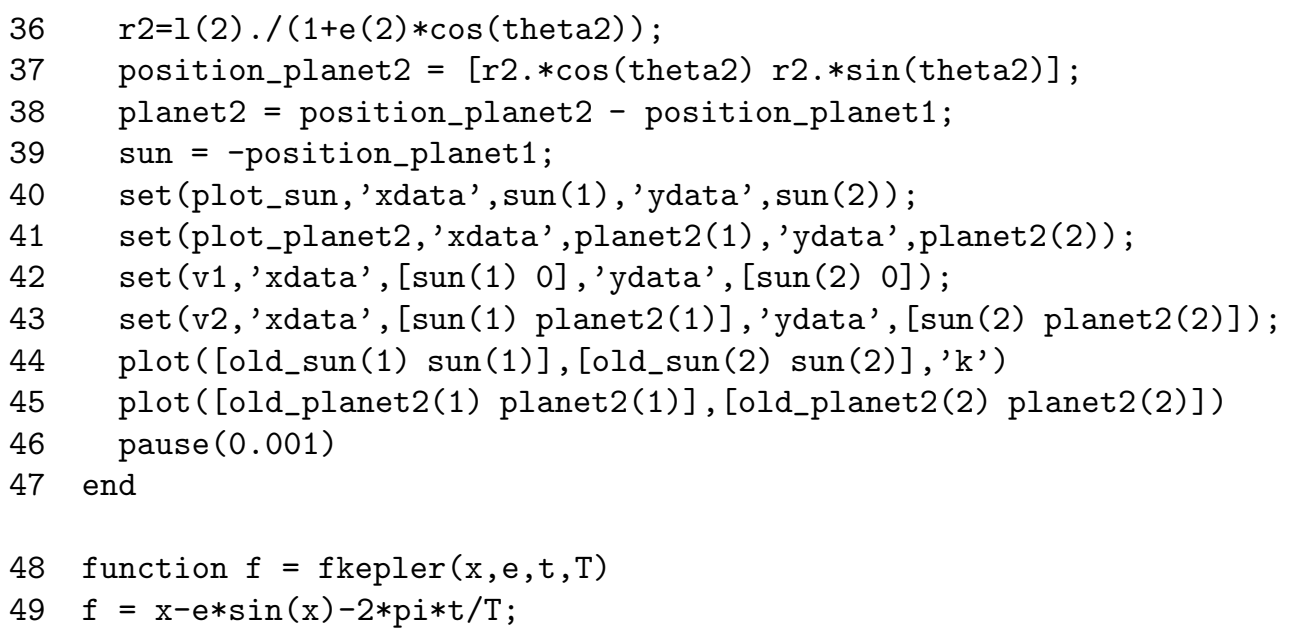

\section{References}

[1] D. Acheson. From Calculus to Chaos. An introduction to dynamics. Oxford University Press.

[2] R.P. Brent. Algorithms for Minimization Without Derivatives. Englewood Cliffs, Prentice-Hall.

[3] C. Kittel, W.D. Knight, and M. Ruderman. Berkeley Physics Course Mechanics vol. 1. McGraw-Hill.

[4] M. Kline, Why Johnny can't add. http://www . marco-learningsystems . com/pages/kline/kline.html.

[5] D.A. Vallado, W.D. Mc Clain. Fundamentals of astrodynamics and applications. McGraw-Hill.

[6] http://www.windows .ucar.edu/ 